\title{
Stereoscopic investigation on plasma density fluctuations in the outer solar corona (Research Note)
}

\author{
D. Telloni ${ }^{1}$, E. Antonucci ${ }^{1}$, S. Dolei ${ }^{2}$, P. Romano ${ }^{2}$, D. Spadaro ${ }^{2}$, and R. Ventura ${ }^{2}$ \\ ${ }^{1}$ National Institute for Astrophysics (INAF), Astrophysical Observatory of Torino, via Osservatorio 20, 10025 Pino Torinese, Italy \\ e-mail: telloni@oato.inaf.it \\ ${ }^{2}$ National Institute for Astrophysics (INAF), Astrophysical Observatory of Catania, via S. Sofia 78, 95123 Catania, Italy \\ Received 1 October 2013 / Accepted 17 February 2014
}

\section{ABSTRACT}

\begin{abstract}
This research note extends a previous work focused on the 2D reconstruction of the spatial distribution and temporal evolution of the plasma density fluctuations in the outer solar corona and based on STEREO COR1-A white-light observations. By using the corresponding total brightness images obtained in the same observational period with the coronagraph COR1-B onboard the "Behind" twin STEREO-B spacecraft, and adopting the same methodological approach as for COR1-A data, it was possible to confirm the results of the previous work and argue for the 3D configuration of the fluctuations of the coronal plasma. This provides further evidence in support of a scenario in which the fluctuating features, which are recurrent and spatially coherent, are localized along the magnetic field lines and points out the crucial role played by the 3D magnetic field topology in the confinement and evolution of the plasma density fluctuations.
\end{abstract}

Key words. methods: data analysis - magnetohydrodynamics (MHD) - Sun: corona - Sun: magnetic fields - Sun: oscillations waves

\section{Introduction}

The observation of wave and oscillatory phenomena at different heights in the solar atmosphere have been vigorously boosted over the past few years by the high spatial resolution and time cadence capabilities of imaging and spectral instruments onboard the SOlar and Heliospheric Observatory (SOHO, Domingo et al. 1995), Transition Region And Coronal Explorer (TRACE, Handy et al. 1999), Solar TErrestrial RElations Observatory (STEREO, Kaiser et al. 2008), and Solar Dynamics Observatory (SDO, Pesnell et al. 2012) satellites.

On the basis of STEREO COR1-A white-light images (Thompson et al. 2003; Howard et al. 2008; Kaiser et al. 2008), Telloni et al. (2013, hereafter Paper I) have very recently shown that the whole solar corona is permeated by large-scale, quasiperiodic intermittent density fluctuations. They are, however, not uniformly distributed, but are instead observed to be clearly structured as either arch-shaped (at low and mid heliographic latitudes) or ray-like (at higher latitudes) features, which are often, though not always, localized along the magnetic field lines, thus appearing as embedded in or shaped by fluctuating magnetic structures. The analysis of the cross-correlation of the fluctuation power along these spatial features, performed in the same paper, has revealed that the plasma fluctuations are highly coherent in phase.

The authors examined in detail two possible interpretations for the detected phenomena. Slow magneto-acoustic waves, stochastically excited by the supergranular convective motions and trapped as standing modes in the closed field lines, might be responsible for the arch-shaped coronal fluctuations. On the other hand, the high degree of coherence of the plasma fluctuations detected along the open magnetic features rules out the possibility that they are produced by outward propagating waves, since they would instead lead the fluctuations to be out of phase along the ray-like structure acting as a channel for the propagating disturbances. Rather, recurrent quasi-periodic magnetic reconnection (due to continuously emerging flux tubes in the polar regions and modulating the intrinsic brightness of the polar ray), as well as any quasi-periodic transverse displacements of the polar rays themselves, might explain the existence of the open fluctuating features.

The results presented in Paper I were achieved by analyzing long-term time sequences of total brightness images obtained with the coronagraph COR1-A and by adopting the wavelet transforms as the diagnostic technique. This method is particularly powerful for analyzing non-stationary time series eventually characterized by variations of power in both time and space. In so doing, the authors were able to map the spatiotemporal evolution of the detected non-stationary variability of the outer corona, by performing a 2D reconstruction of the total brightness variance. The simultaneous data coming from the coronagraph COR1 onboard the "Behind" STEREO-B spacecraft have not been examined in that context.

This research note supports and complements the results of the previous work by exploiting the stereoscopic observational capabilities of the twin STEREO spacecrafts. The COR1-B time series of total brightness images, which refers to the same time interval as in Paper I, was analyzed by the wavelet transforms and the results combined with those previously obtained for COR1-A, in order to stereoscopically investigate the structure of the plasma density fluctuations. The two different vantage points provided by STEREO observations indeed make it possible to obtain useful hints on the 3D spatial pattern of the detected fluctuating coronal features and to cast further light on 


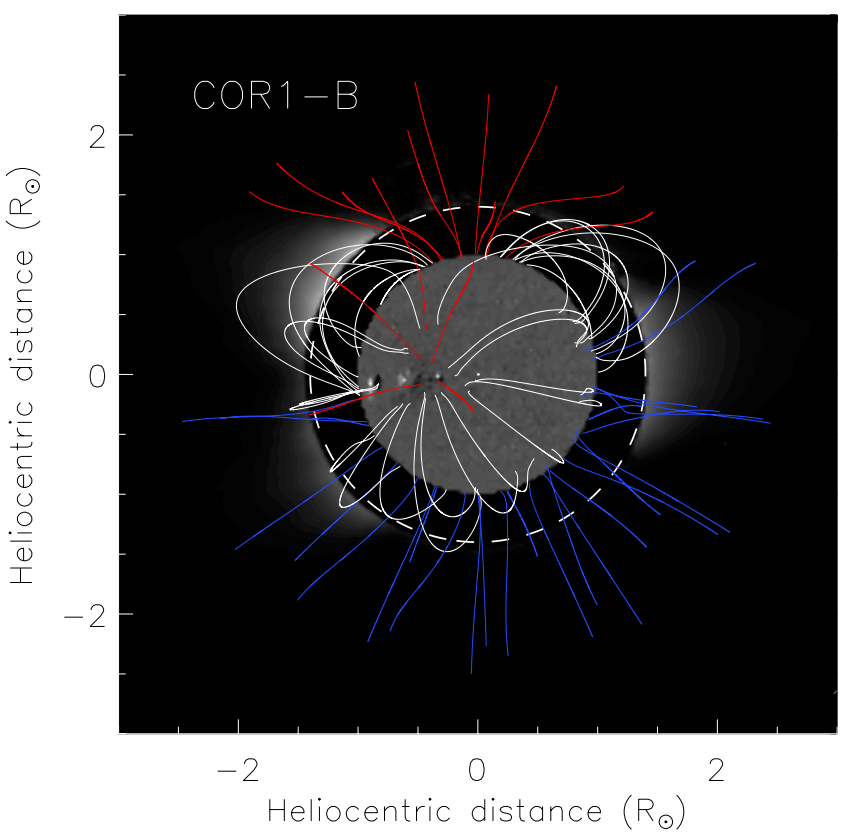

Mean solar brightness (arbitrary units)

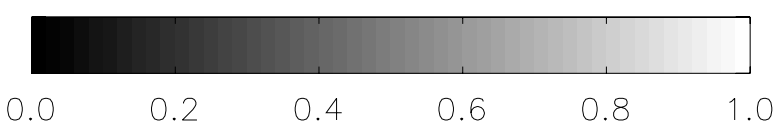

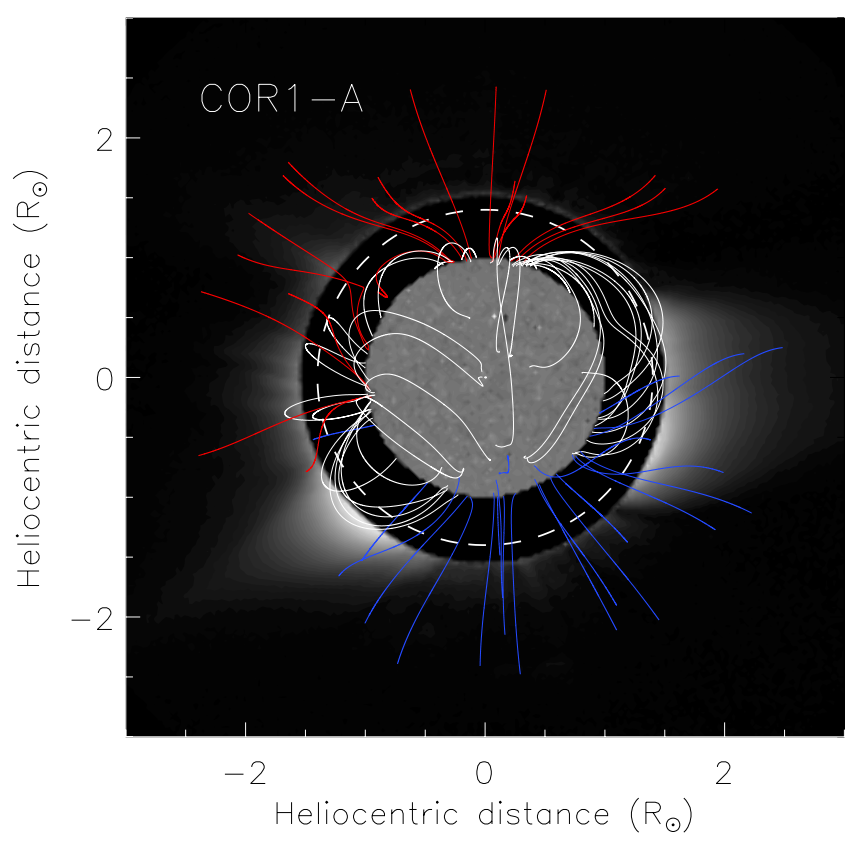

Mean solar brightness (arbitrary units)

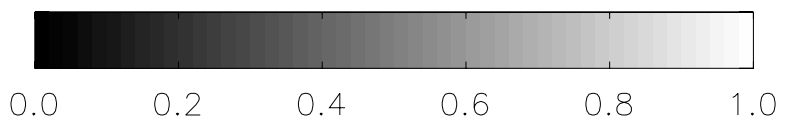

Fig. 1. Total brightness images of the outer solar corona observed by COR1-B (left panel) and COR1-A (right panel) coronagraphs on April 18, 2008 at 21:50 UT, from 1.4 to $4.0 R_{\odot}$; the extrapolated magnetic field lines (white lines refer to closed field lines, red and blue lines to open field lines, with positive and negative polarity, respectively) and the SOHO/MDI disk magnetograms as seen from the STEREO-B and STEREO-A viewpoints on April 19, 2008 at 00:04 UT are also shown; the white dashed lines indicate the edge of the STEREO occulter.

the correlation between coronal periodic and quasi-periodic phenomena and the 3D magnetic field topology.

The layout of the note is as follows. The description of the observations and of the method of analysis is given in Sects. 2 and 3 provides the presentation of the results achieved by applying the wavelet technique on the STEREO data. Discussion of the results and conclusions are given in Sect. 4.

\section{Observations and data analysis}

The analysis presented in this note was performed on long temporal sequences of total brightness images of the outer solar corona, simultaneously sampled at five-minute time cadence, by the two coronagraphs COR1-A and COR1-B during the minimum activity of solar cycle 24, from April 14, 2008 at 00:05 UT to April 22, 2008 at 23:55 UT. At that epoch the angular separation of the two satellites was $48.4^{\circ}$. Both the coronagraphs provide $1024 \times 1024$ pixel off-limb coronal imaging from 1.4 to $4.0 R_{\odot}$, with a spatial resolution of $7.5 \mathrm{arcsec}$ per pixel. The total brightness is derived by combining the images recorded at three polarizer positions separated by $120^{\circ}$ (Billings 1966).

Figure 1 shows, as an example, the total brightness of the solar corona on April 18, 2008 at 21:50 UT as observed by COR1-B (left panel) and COR1-A (right panel) from their respective viewpoints. The SOHO/MDI (Michelson Doppler Imager, Scherrer et al. 1995) disk magnetograms (as seen from the STEREO-B and STEREO-A satellites on April 19, 2008 at 00:04 UT) have been superimposed on the corresponding coronal white-light images. Furthermore, the coronal magnetic field lines, extrapolated via the potential-field source-surface (PFSS, Schrijver \& DeRosa 2003) approximation, are outlined in both the COR1-B and COR1-A total brightness maps, by properly superimposing the instantaneous orthographic projection of the modeled 3D coronal magnetic configuration as viewed by the corresponding perspective of the STEREO satellites on each white-light image. The coronal magnetic field was calculated from SOHO/MDI photospheric field observations, by assuming that the field is potential in the coronal volume between the photosphere and a spherical source surface located at a height of $2.5 R_{\odot}$. At that height the field is forced to be radial to approximate the effect of the accelerating solar wind on the field configuration.

Two coronal streamers are present in the COR1-A total brightness image (at the southeastern and western limbs). Two streamers (at the northeastern and western limbs) are present in the COR1-B image as well. The equatorial streamer observed at the western limb by both the coronagraphs appears to protrude to lower heights in the COR1-B image with respect to the image of COR1-A. This probably indicates that this structure lies closer to the plane of the sky (POS) of COR1-A than to that of COR1-B. The modeled closed magnetic field lines outline the coronal streamers observed by both coronagraphs with good approximation.

Following the methodological approach adopted in Paper I for COR1-A data, the variance of the coronal total brightness obtained by analyzing COR1-B white-light images is mapped. The reader is referred to Paper I for more exhaustive details on the analysis methodology. For sake of clarity, it is only recalled here that the wavelet transforms are able to decompose a signal into the time-frequency space (Torrence \& Compo 1998), thus obtaining the temporal modulation of the power at any given 

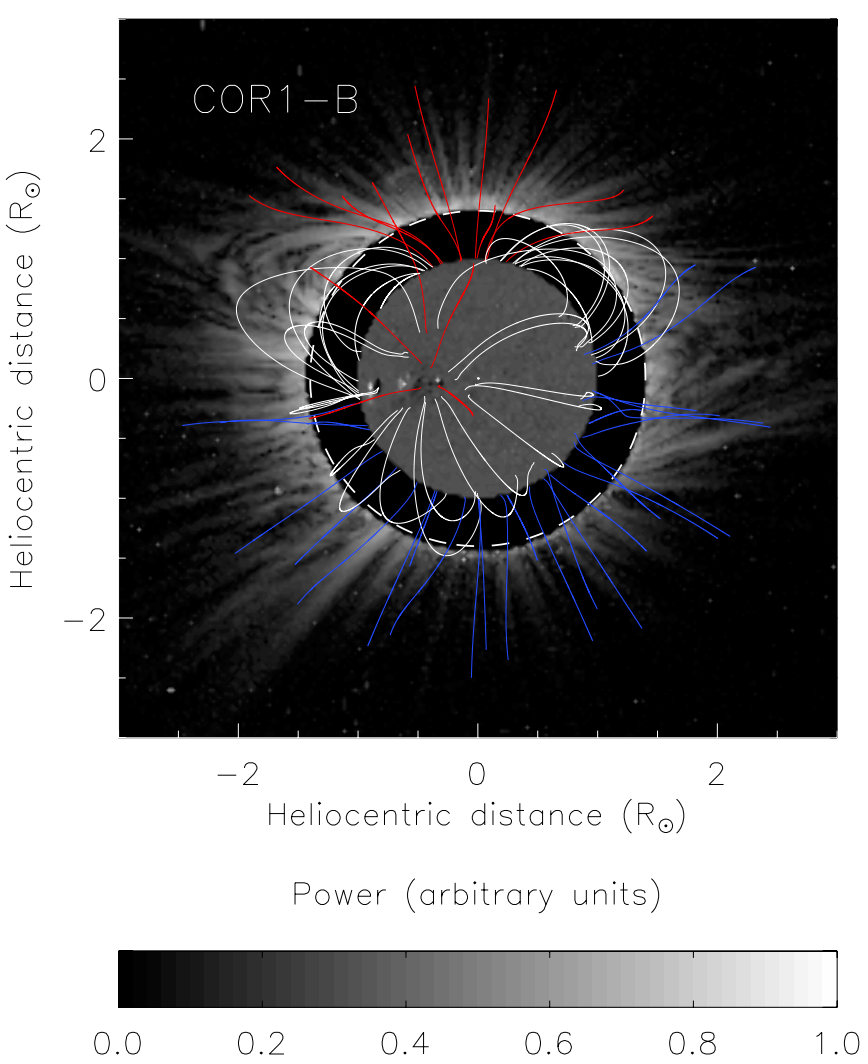

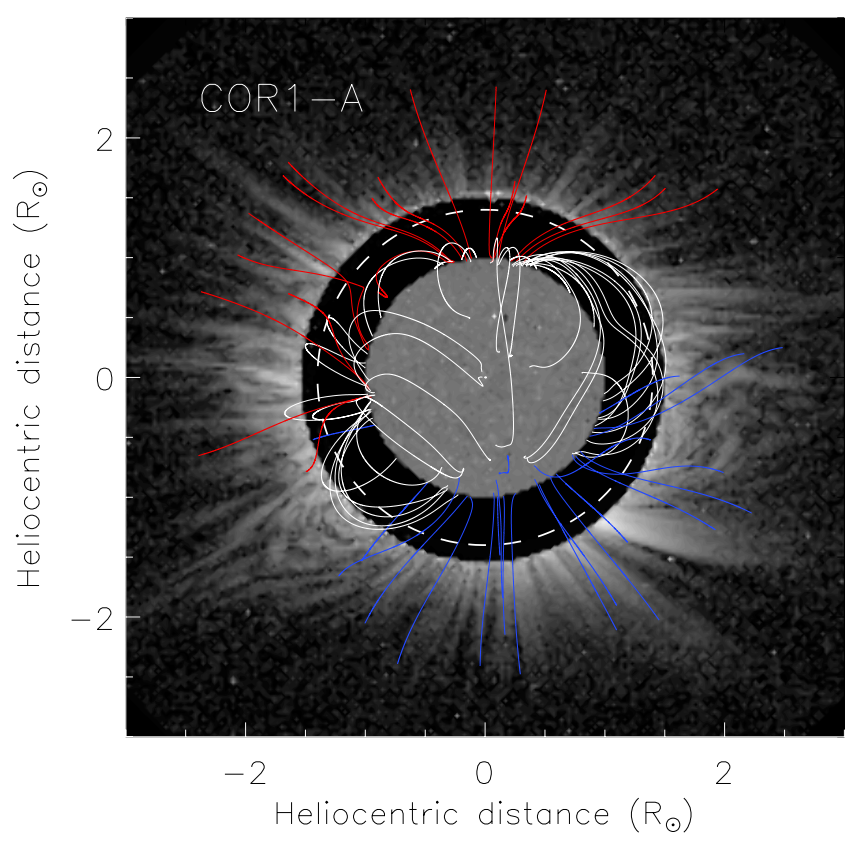

Power (arbitrary units)

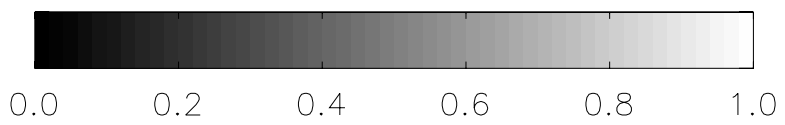

Fig. 2. 2D maps of the power of the plasma density fluctuations, over the 4-8 h periodicity band, derived in the fields of view of COR1-B (left panel) and COR1-A (right panel), on April 18, 2008 at 21:50 UT; the power of the fluctuating structures is significant with a $99 \%$ statistical confidence, for a white-noise process; the extrapolated magnetic field lines (white lines refer to closed field lines, red and blue lines to open field lines, with positive and negative polarity, respectively) and the SOHO/MDI disk magnetograms as seen from the STEREO-B and STEREO-A viewpoints on April 19, 2008 at 00:04 UT are also shown; the white dashed lines indicate the edge of the STEREO occulter.

timescale. It turns out that the spatiotemporal evolution of the power of the fluctuating coronal features detected over a certain range of timescales can be reconstructed by combining into a $2 \mathrm{D}$ contour plot the results achieved pixel by pixel of the coronagraphic images. Since in Paper I the authors found that the strongest fluctuations and the dominant mode of variability have timescales in the range of $4-8 \mathrm{~h}$, the present analysis focuses on this periodicity band.

\section{Results}

The left- and righthand panels of Fig. 2 report, as an example, the $2 \mathrm{D}$ maps of the wavelet power in the $4-8 \mathrm{~h}$ periodicity band as derived from COR1-B and COR1-A observations, respectively, on April 18, 2008 at 21:50 UT (the same instant as in Fig. 1). The power plotted exceeds the $99 \%$ confidence level determined via a 2 degree-of-freedom $\chi^{2}$ distribution (see Torrence \& Compo 1998, and references therein). The identifiable features thus present in the field of view (FOV) of both coronagraphs are significant with respect to the background level of random fluctuations of plasma density in the quiet solar corona. The SOHO/MDI disk magnetograms and the extrapolated magnetic field lines, as seen from the STEREO-B and STEREO-A perspectives on April 19, 2008 at 00:04 UT, are superimposed on the 2D maps.

Strong fluctuating features, with approximately the same power and similar characteristic transverse and longitudinal spatial scales, are revealed in the FOV of both COR1-A and COR1-B coronagraphs as arch-shaped (at low and mid latitudes) and ray-like (at higher latitudes) structures, extending up to about $2 R_{\odot}$. In particular, systems of two or more encapsulated closed fluctuating arches, separated by dark cavities, are evident and appear to be embedded in the streamer structures observed by COR1-A and COR1-B (Fig. 1). For both coronagraphs, the thickness $d_{\perp}$ and the length $L$ (extrapolated down to the solar disk) of the open and closed power features are $d_{\perp} \sim 0.01 R_{\odot}=$ $7 \mathrm{Mm}$ and $L \sim 1.4-2.9 R_{\odot}=0.98-2.03 \times 10^{3} \mathrm{Mm}$, respectively (Paper I). Some high-power fluctuating structures, which do not have a resolved counterpart in the white-light images of the solar corona, clearly outline the lines of the coronal magnetic field (Fig. 2), thus appearing as confined by the magnetic field topology. Analogous results were obtained for all the frames referring to the whole observational time interval.

\section{Discussion and conclusions}

The comparative study carried out in the present research note strongly supports the results presented in Paper I. The maps of the variance of the coronal brightness obtained for both COR1-A and COR1-B data clearly outline 2D non-stationary fluctuating power features characterized by similar power level and spatial scales, and localized along the magnetic field lines, thus confirming and strengthening the findings of a tight correlation between coronal periodic or quasi-periodic oscillatory processes and the coronal magnetic field, as pointed out in Paper I.

Moreover, the stereoscopic comparison between the two different STEREO coronagraphic viewpoints allowed us to argue for the $3 \mathrm{D}$ nature of the detected fluctuations, whose pattern 
appears to be structured and confined by the magnetic field topology. Projecting the 3D coronal magnetic field configuration obtained by the PFSS extrapolation on the corresponding perspectives of the two coronagraphs, we get a satisfactory overlapping of the magnetic field lines with the fluctuation power concentrations both in COR1-A and COR1-B maps.

Interestingly, the closed arch-shaped power structures are much more evident in the coronagraphic images of COR1-A where the streamers, particularly the equatorial one identified at the western limb in both total brightness images, are very likely lying closer to the POS of the "Ahead" coronagraph, as might be expected if the fluctuating features were part of the streamer structures themselves (Sect. 2). Conversely, the open ray-like structures identified in the polar regions of the $2 \mathrm{D}$ maps of the plasma density fluctuation power appear very similar for both coronagraphs, since polar structures are characterized by similar longitudinal extension, independently of the viewpoints.

It turns out that the diagnostic technique adopted in this note more efficiently identifies high-power fluctuating features when the coronal plasma structures, in which the fluctuations are embedded, lie close to the POS of the coronagraphic image. A tilt of the fluctuating structures relative to the POS would therefore produce a variable column depth along the line of sight (LOS), thus modulating the power estimated by means of the wavelet transforms. This might represent a strong clue on the 3D spatial pattern of the plasma density fluctuations.

In Paper I the arch-shaped power enhancements were interpreted as due to intrinsic coronal variability, since any other effect that could in principle mimic plasma density fluctuations, such as pulses of material or ions injected in the arch and trapped in the flux tube to bounce back and forth along the magnetic structure, has been ruled out on the basis of the high coherence of the fluctuations detected along the arch-shaped feature. Indeed, the non-intrinsic variability driven by such effects would be out of phase along the closed structure. In Paper I it was proposed that slow magneto-acoustic standing waves are the ideal candidates for interpreting the plasma fluctuations embedded in the closed structures, since both timescales and lengths of the fluctuating arch-shaped features are compatible with the period and the wavelength of a Magnetohydrodynamic (MHD) slow-mode wave, which, triggered by pressure disturbances generated near both footpoints and propagating in opposite directions along the magnetic flux tube would thus remain trapped in the arch structure as a standing wave. Furthermore, the speed of such an acoustic standing wave $\frac{2 L}{T} \approx 140-280 \mathrm{~km} \mathrm{~s}^{-1}$, where $L \sim 2.9 R_{\odot}=2.03 \times 10^{3} \mathrm{Mm}$ and $T=4-8 \mathrm{~h}$ are the characteristic temporal and length scales of the fluctuating arch-shaped structure, is in good agreement with the phase speed of a slow MHD wave, which, for typical coronal temperatures of about $1.5 \mathrm{MK}$, is close to the sound speed $c_{s}=200 \mathrm{~km} \mathrm{~s}^{-1}$ (e.g., Priest 1987). The observational evidence that the lengths of the arches embedding density fluctuations with periods in the range of $4-8 \mathrm{~h}$ have the same order of magnitude when revealed in the 2D maps of both coronagraphs, provides a clear indication that the arch length itself acts as a filter of slow MHD standing waves with the appropriate wavenumber.

The main results of this research note can be summarized as follows. i) The density fluctuations are embedded in arch-shaped or ray-like non-stationary features. ii) The 3D configuration inferred for the fluctuating power structures appears as guided and regulated by the topology of the coronal magnetic field. iii) The non-stationary features sustain just those MHD waves that have wavelengths compatible with their characteristic temporal and spatial scales, which therefore turn out to be the key parameters in the MHD activity of the solar corona.

The $3 \mathrm{D}$ reconstruction of a variety of coronal mass ejections (CMEs) observed by STEREO has been carried out in the past years by adopting different methods (e.g., Mierla et al. 2009, 2011; Moran \& Davila 2004; Thernisien et al. 2006), all based on an ad hoc code optimized for reconstructing the bubble-like shape of the CMEs and the propagation direction of such very dynamic phenomena. The development of a code specifically devoted to reconstruct the 3D morphology of the fluctuating wavelet power structures might be the task of a future work.

Acknowledgements. This work was supported by the Italian Space Agency (ASI) grants (I/013/12/0). The research leading to these results has received funding from the European Commission's Seventh Framework Program under the grant agreements eHEROES (project 284461). The authors thank the anonymous referee for the precious comments and suggestions that contributed to improving the paper.

\section{References}

Billings, D. E. 1966, A guide to the solar corona (New York: Academic Press) Domingo, V., Fleck, B., \& Poland, A. I. 1995, Sol. Phys., 162, 1

Handy, B. N., Acton, L. W., Kankelborg, C. C., et al. 1999, Sol. Phys., 187, 229

Howard, R. A., Moses, J. D., Vourlidas, A., et al. 2008, Space Sci. Rev., 136, 67

Kaiser, M. L., Kucera, T. A., Davila, J. M., et al. 2008, Space Sci. Rev., 136, 5

Mierla, M., Inhester, B., Marqué, C., et al. 2009, Sol. Phys., 259, 123

Mierla, M., Chifu, I., Inhester, B., Rodriguez, L., \& Zhukov, A. 2011, A\&A, 530, L1

Moran, T. G., \& Davila, J. M. 2004, Science, 306, 66

Priest, E. R. 1987, Solar Magneto-hydrodynamics (Dordrecht: D. Reidel)

Pesnell, W. D., Thompson, B. J., \& Chamberlin, P. C., et al. 2012, Sol. Phys., 275,3

Scherrer, P. H., Bogart, R. S., Bush, R. I., et al. 1995, Sol. Phys., 162, 129

Schrijver, C. J., \& DeRosa, M. L. 2003, Sol. Phys., 212, 165

Telloni, D., Ventura, R., Romano, P., Spadaro, D., \& Antonucci, E. 2013, ApJ, 767, 138

Thernisien, A. F. R., Howard, R. A., \& Vourlidas, A. 2006, ApJ, 652, 763

Thompson, W. T., Davila, J. M., Fisher, R. R., et al. 2003, Proc. SPIE, 4853, 1

Torrence, C., \& Compo, G. P. 1998, Bull. Am. Meteorol. Soc., 79, 61 